\title{
Espécies novas de Tomosvaryella Aczél (Diptera, Pipunculidae) da Argentina e chave para as espécies sul-americanas
}

\author{
Rosaly Ale-Rocha ${ }^{1} \&$ Bruna Barbosa de Souza ${ }^{1,2}$
}

${ }^{1}$ Coordenação de Pesquisas em Entomologia, Instituto Nacional de Pesquisas da Amazônia, Caixa Postal 478, 69011-970 Manaus-AM, Brasil. ${ }^{2}$ Bolsista de Iniciação Científica FAPEAM. bruna_fpf@hotmail.com

\begin{abstract}
New species of Tomosvaryella Aczél (Diptera, Pipunculidae) from Argentina and key to the South American species. Two new species of Tomosvaryella are described from Argentina, Tomosvaryella lobata sp. nov. and Tomosvaryella platypoda sp. nov.; an identification key is presented for the South American species of Tomosvaryella and new records of Tomosvaryella species to Brazil and Argentina are provided.
\end{abstract}

KEYWORDS. Description; distribution; key; new species.

RESUMO. Espécies novas de Tomosvaryella Aczél (Diptera, Pipunculidae) da Argentina e chave para as espécies Sul-americanas. Duas espécies novas de Tomosvaryella Aczél são descritas para a Argentina, Tomosvaryella lobata sp. nov. e Tomosvaryella platypoda sp. nov.; uma chave de identificação para as espécies sul-americanas de Tomosvaryella é apresentada e são fornecidos registros novos de espécies de Tomosvaryella para o Brasil e Argentina.

PALAVRAS-CHAVE. Chave; descrição; distribuição; espécies novas.

O gênero Tomosvaryella é cosmopolita, engloba cerca de 280 espécies muito semelhantes entre si, a maioria separada apenas por caracteres da terminália e trocanter posterior dos machos. Os espécimes podem ocorrer em grande número em ambientes com vegetação predominante de gramíneas onde os hospedeiros são mais freqüentes. O relacionamento das espécies de Tomosvaryella com o hospedeiro ainda não é satisfatoriamente conhecido, mas, até o momento, os dados evidenciam uma associação exclusiva com Cicadellidae, Deltocephalinae (Hemiptera) (De Meyer 1989).

As espécies incluídas em Tomosvaryella são caracterizadas pela ausência de cerdas propleurais, pterostigma e veia M2 e deslocamento da veia r-m para o meio ou próximo ao meio da seção superior da célula dm. Formam um grupo distintamente monofilético (Rafael \& De Meyer 1992).

As espécies neotropicais foram revisadas por Ale-Rocha (1992a, b, 1993, 1996) e Ale-Rocha \& Rafael (1995). Uma chave de identificação para as espécies do México, América Central e Antilhas está disponível em Ale-Rocha \& Rafael (1995). Atualmente, 30 espécies são conhecidas para a Região Neotropical (De Meyer 1996; De Meyer \& Skevington 2000).

Neste trabalho são descritas duas espécies novas de Tomosvaryella para a Argentina, elevando para 13 o número de espécies conhecidas para o país (sendo sete endêmicas); são registradas novas ocorrências para a América do Sul e é apresentada uma chave de identificação para as espécies deste continente.

\section{MATERIAL E MÉTODOS}

O material estudado faz parte do acervo da Coleção de Invertebrados do Instituto Nacional de Pesquisas da Amazônia - INPA, Manaus, Brasil; e do Planta Piloto de Procesos Industriales Microbiológicos - PROIMI, San Miguel de Tucumán, Argentina. Os espécimes estão conservados a seco e montados em triângulo de papel.

Para estudo da terminália masculina, o abdômen foi cortado na altura do terceiro segmento e a parte destacada foi tratada com ácido lático $80 \%$ a quente por cerca de $15 \mathrm{mi}$ nutos. Posteriormente, o material foi guardado em microtubos de plástico contendo glicerina e fixado junto ao espécime correspondente. A terminologia utilizada nas descrições segue McAlpine (1981) e Skevington \& Yeates (2001).

As informações apresentadas entre colchetes em Material Examinado são dados complementares ausentes nas etiquetas. Estados/províncias e países assinalados com asterisco representam novos registros de distribuição geográfica.

As figuras das espécies já descritas que ilustram a chave de identificação (Figs. 1-98) foram retiradas de diferentes publicações e tiveram seu tamanho alterado para se adequarem ao tamanho da prancha desta revista. Devido ao grande número de figuras em cada prancha, a repetição da escala de cada figura individualmente resultaria em uma prancha visualmente muito confusa. Portanto, optamos por suprimir essas escalas que podem ser obtidas nos trabalhos originais citados nas legendas. 


\section{RESULTADOS}

\section{Tomosvaryella amazonensis De Meyer \& Skevington, 2000}

(Figs. 1, 2, 34, 35)

Tomosvaryella amazonensis De Meyer \& Skevington, 2000: 8

Tomosvaryella pulchra Ale-Rocha, 1992a: 347 (pré-ocupado Kozánek 1992).

Diagnose. Tarsômeros castanhos; trocanter posterior com duas protuberâncias espiniformes ventrais (Figs. 1, 2); tarso posterior levemente dilatado; sintergoesternito 8 com duas protuberâncias ventrais (Fig. 35); surstilos subsimétricos com protuberância dorso-basal (Fig. 34).

Material examinado: ARGENT[ina], Salta, Tacuil, 01-15.ix.1969, Terán, Willink \& Stange, Malaise, 9 machos (3 INPA, 6 PROIMI); Tawil, 01-15.viii.1969, 1 macho (PROIMI); Yacochuia, 01-15.i.1969, 2 machos (PROIMI); 16-31.iii.1969, 1 macho (PROIMI); 01-15.xii.1970, 1 macho (PROIMI); Catamarca, L. Nacimiento, Malaise, 15-28.ii.1968, Terán, Willink \& Stange, 11 machos (4 INPA, 7 PROIMI); 01-15.iii.1968, 14 machos (4 INPA, 10 PROIMI); 15-30.xi.1968, 18 machos (3 INPA, 15 PROIMI); 01-15.iv.1969, 2 machos (PROIMI); Tucumán, Tapia, Malaise, 01-15.ii.1993, Cuezzo \& Fidalgo, 7 machos (2 INPA, 5 PROIMI); Belen, 16-31.x.1968, 3 machos (PROIMI).

Distribuição geográfica: Brasil, Chile, Paraguai e Argentina (Santiago Del Estero, Salta*, Catamarca*, Tucumán*).

\section{Tomosvaryella aurata Ale-Rocha, 1996}

(Figs. 3, 4, 36, 37)

Tomosvaryella aurata Ale-Rocha, 1996: 166, figs. 1-8; De Meyer \& Skevington, 2000: 8 (catálogo).

Diagnose. Laterotergito com pruinosidade dourada; abdome largo com manchas laterais de pruinosidade cinza-prateada; trocanter posterior com espinho longo e delgado (Fig. 4); $5^{\circ}$ tarsômero anterior com projeção basal na face ventral (Fig. 3); surstilos subsimétricos (Figs. 36, 37); ápice do guia fálico e ramificações do edeago curtas; bulbo do apódema ejaculador com tubérculos.

Material examinado: BRASIL, Amazonas, Rio Jaú, Meriti, Mun[icípio] Novo Airão, 1 macho (INPA).

Distribuição geográfica: Argentina, Brasil* (Amazonas)

\section{Tomosvaryella bissulca Ale-Rocha, 1996}

(Figs. 6, 39-42)

Tomosvaryella bissulca Ale-Rocha, 1996: 167, figs. 9-15; De Meyer \& Skevington, 2000: 8 (catálogo).

Diagnose. Tórax com cerdas desenvolvidas; fêmur posterior com cerdas alongadas na face posterior; trocanter posterior com protuberância ventral achatada (Fig. 6); tarsos variando do castanho dorsalmente com a face ventral amare- la (espécimes do Chile) ou amarelos com o último tarsômero marrom (espécimes da Argentina); sintergoesternito 8 alongado (Figs. 40, 42); surstilos longos e robustos com ápices curvados para dentro (Fig. 41); guia fálico com espinho dorsal bifurcado apicalmente (Fig. 39); bulbo do apódema ejaculador longo e estreito.

Material examinado: ARGENT[ina], Salta, Tacuil, 01-15.ix.1969, Terán, Willink \& Stange, Malaise, 3 machos (1 INPA, 2 PROIMI); Catamarca, L. Nacimiento, 15-28.ii.1968, 3 machos (1 INPA, 2 PROIMI); 15-30.xi.1968, 7 machos (3 INPA, 4 PROIMI).

Distribuição geográfica: Chile, Argentina* (Salta, Catamarca).

\section{Tomosvaryella crinita Ale-Rocha, 1996}

(Figs. 8, 9, 46, 47)

Tomosvaryella crinita Ale-Rocha, 1996: 169, figs. 25-32; De Meyer \& Skevington, 2000: 8 (catálogo).

Diagnose. Tarsos amarelos; trocanter posterior com protuberância ventral arredondada (Fig. 9); tarsos posteriores alargados com franja de cerdas longas na face dorsal (Fig. 8); surstilos subsimétricos, longos (Figs. 46, 47); bulbo do apódema ejaculador alongado.

Material examinado: ARGENT[ina], Salta, Tacuil, 16-31.i.1969, Téran, Willink \& Stange, Malaise, 1 macho (PROIMI); Catamarca, L. Nacimiento, 01-15.iii.1968, Téran, Willink \& Stange, Malaise, 1 macho (PROIMI); 1530.xi.1968, 1 macho (INPA); 01-15.iv.1969, 1 macho (INPA).

Distribuição geográfica: Argentina (Santiago Del Estero, Salta*, Catamarca*).

\section{Tomosvaryella curta Ale-Rocha, 1996}

(Figs. 48-49)

Tomosvaryella curta Ale-Rocha, 1996: 170, figs. 33-40; De Meyer \& Skevington, 2000: 8 (catálogo).

Diagnose. Tórax com cerdas desenvolvidas; fêmur posterior com cerdas alongadas na face posterior; surstilos curtos e assimétricos, o esquerdo com ápice truncado (Figs. 48, 49); cercos alongados; guia fálico com ápice longo.

Material examinado: ARGENT[ina], [Buenos Aires], Canuela, Trampa, Malaise, ix.1994-v.1995, Terán Willink \& Stange, 1 macho (PROIMI).

Distribuição geográfica: Chile, Argentina* (Buenos Aires).

\section{Tomosvaryella diffusa Ale-Rocha, 1996}

(Figs. 10, 11, 50, 51)

Tomosvaryella diffusa Ale-Rocha, 1996: 169, figs. 41-49; De Meyer \& Skevington, 2000: 8 (catálogo).

Diagnose. Tarsos posteriores alargados (Fig. 11); trocanter posterior com protuberância grande ventral (Fig. 10); surstilos 

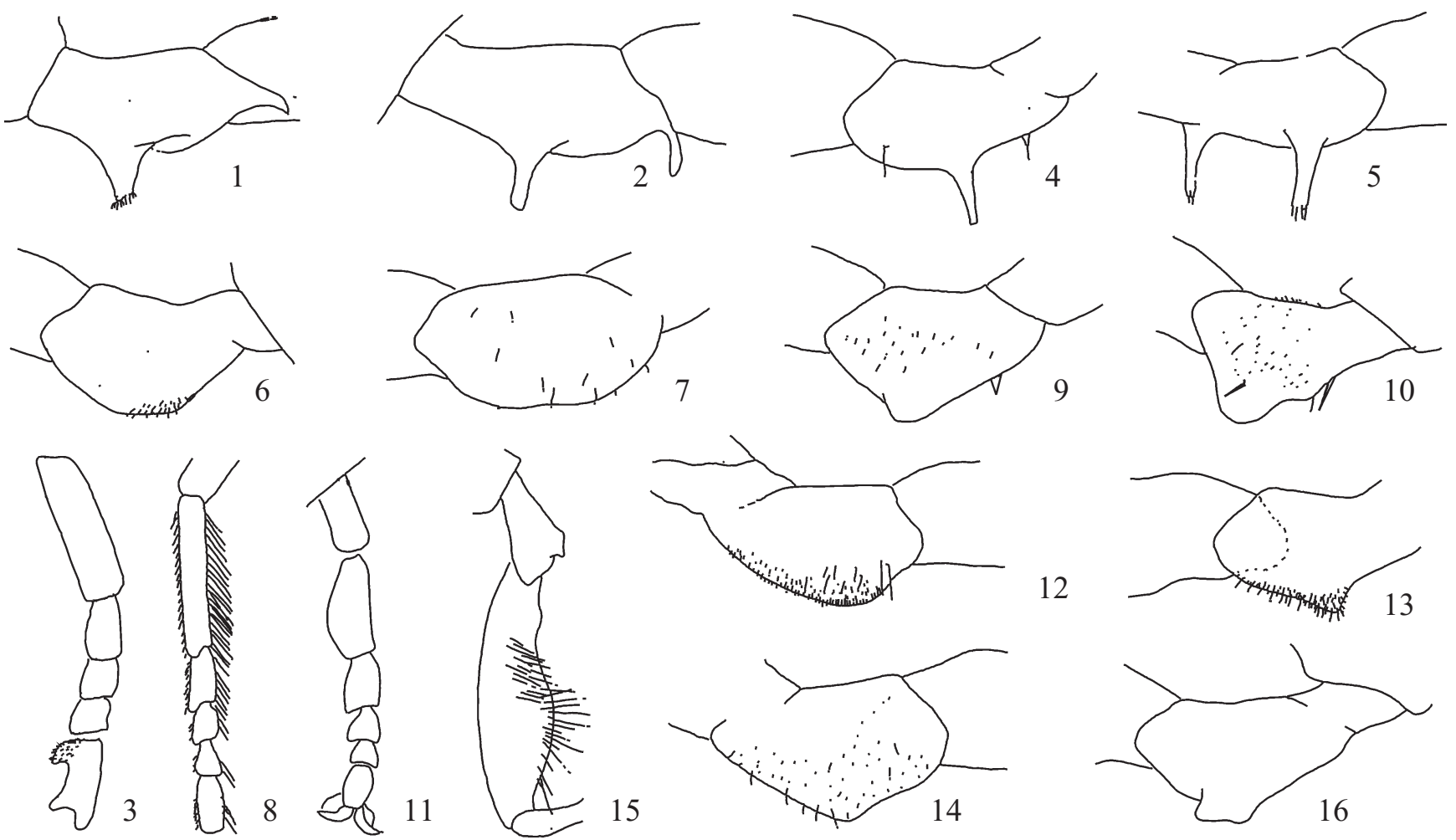

Figs. 1-16. Trocanter posterior (Figs. 1-2, 4-7, 9, 10, 12-16) e tarsos posteriores (Figs. 3, 8, 11, 15): 1-2, Tomosvaryella amazonensis; 3-4, T. aurata; 5, T. bidens; 6, T. bissulca; 7, T. chilensis; 8-9, T. crinita; 10-11, T. diffusa; 12, T. ekyphysis; 13, T. galapagensis; 14, T. incompta; 15-16, T. longiseta. (Figuras retiradas de Ale-Rocha 1992a, b, 1993, 1996 e Ale-Rocha \& Rafael 1995).

alongados, assimétricos, arqueados em vista lateral (Figs. 50, 51); ápice do guia fálico curto.

Material examinado: BRASIL, D[istrito] F[ederal], Brasília, Res[erva] Biol[ógica], IBGE, 1555'58"S 4751'02"W, 09-16.x.1981, A. B. F. Dias et al, 2 machos (INPA); 25.xi.1982, 1 macho (INPA); ARGENT[ina] Salta, Yacochuia, Malaise, 01-15.x.1968, Terán Willink \& Stange, 2 machos (1 INPA, 1 PROIMI); 01-15xii.1968, 2 machos (PROIMI); 01-15.xii.1970, 1 macho (INPA).

Distribuição geográfica: Guiana, Brasil (Distrito Federal*), Argentina (Santiago Del Estero, Salta*).

\section{Tomosvaryella lobata sp. nov.}

(Figs. 99-104)

Diagnose. Trocanter posterior com protuberância ventral pré-apical grande, longa e curvada para a base; surstilos subsimétricos, com as faces internas voltadas para o dorso na metade distal; guia fálico com ápice alongado; tarsos delgados, $1^{\circ}$ tarsômero posterior densamente piloso ventralmente.

Descrição. Holótipo macho. Corpo: 3,5 mm Asa: 2,8 mm. Cabeça: pós-crânio marrom-escuro a preto com pruinosidade cinza; triângulo ocelar marrom a preto; ocelos marrons; olhos unidos na fronte por distância equivalente a 7-8 facetas; triângulo frontal largo com pruinosidade cinza-prateada; escapo marrom-escuro; pedicelo marrom-escuro com pequenas cerdas pretas no ápice; flagelo amarelo com ápice filiforme quase tão longo quanto a sua base; face marrom-escura a preta com pruinosidade cinza-prateada, visível a certa luz. Tórax: escuto marrom-escuro a preto com faixa basal de pruína cinza mais densa; cerdas castanho-claras, conspícuas, discretamente alongadas na região pré-sutural; lobo póspronotal amarelo-pálido com cerdas amarelas; calo pós-alar marrom-escuro a preto com pruinosidade cinza; mesopleura castanho-clara. Pernas: coxas marrons, anterior com ápice amarelo; coxa média com cerdas castanhas no ápice; trocanter anterior amarelo; trocanteres médio e posterior marrons com ápice amarelo; trocanter posterior com protuberância alargada, longa e curvada para base (Fig. 103), $1^{\circ}$ tarsômero posterior densamente piloso ventralmente (Fig. 104); fêmures marrom-escuros com ápices amarelos; cerdas ventrais presentes na base do fêmur mediano; ctenídios ventrais curtos presentes na metade distal dos fêmures anterior e médio; fêmur posterior com cerdas longas, delgadas, amarelas e esparsas na metade distal; tíbias marrons com 1/3 basal e ápice amarelos; tarsos amarelos com cerdas amarelas brilhantes; $1^{\circ}$ tarsômero anterior e médio duas vezes mais longo que o $2^{\circ}$; tarsos posteriores pouco alargados. Abdômen: oval, alongado; tergitos marrom-escuros a pretos com cerdas castanhas dispersas; tergito 1 com tufo de cerdas amarelas nas laterais, marrom-claro na base e com uma faixa estreita mediana de pruína cinza; manchas de pruinosidade cinza mais fracas nas laterais dos segmentos 2-5. Asa: hialina; $3^{\mathrm{a}}$ seção costal do mesmo comprimento da 4; ; lobo anal largo; base da asa com tufo de cerdas amarelas; halter amarelo-pálido. 

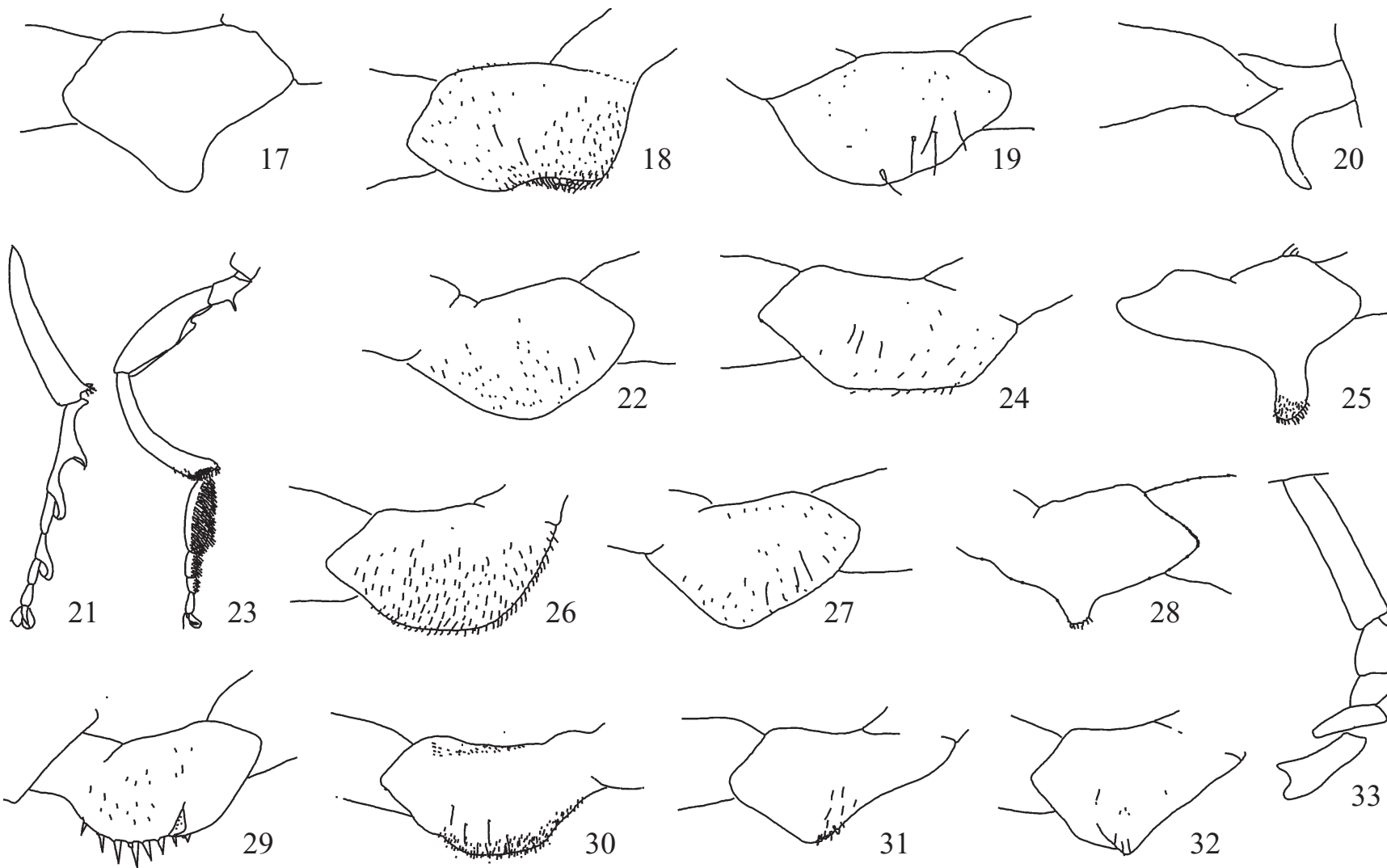

Figs. 17-33. Trocanter posterior (Figs. 17-20, 22, 24-32), pernas posteriores (Figs. 21 e 23) e tarsômeros anteriores (Fig. 33): 17, Tomosvaryella lynchi; 18, T. manauensis; 19, T. mediocris; 20-21, T. ornatitarsalis; 22, T. pectinalis; 23, T. perissosceles; 24, T. platensis; 25, T. prostata; 26, T. santiagoensis; 27, T. scopulata; 28, T. spangleri; 29, T. spinosa; 30, T. subvirescens; 31, T. venezuelana; 32-33, T. virlai. (Figuras retiradas de Ale-Rocha $1992 \mathrm{a}$, b, 1993 , 1996 e Ale-Rocha \& Rafael 1995).

Terminália: sintergoesternito 8 menor que o tergito 5 e área membranosa curta na face ventral (Fig. 99); surstilos curtos, amarelos, subsimétricos, as faces internas voltadas para o dorso na metade distal (Figs. 99, 101); guia fálico com ápice alongado (Fig. 102).

Fêmea. Desconhecida.

Material examinado: Holótipo Macho (PROIMI), ARGENT[INA], Salta, Tacuil, 01-15.ix.1969, Terán, Willink \& Stange, Malaise. Parátipos: ARGENT[INA], Salta, Tacuil, 01-15.ix.1969, Terán, Willink \& Stange. Malaise, 2 machos (1 INPA, 1 PROIMI)

Distribuição geográfica: Argentina (Salta).

Etimologia. Do latim lobus = projeção alongada, protuberância. Refere-se à grande projeção ventral no trocanter posterior do macho.

\section{Tomosvaryella longiseta Ale-Rocha, 1996}

(Figs. 15, 16, 59-61)

Tomosvaryella longiseta Ale-Rocha, 1996: 169, figs. 66-75; De Meyer \& Skevington, 2000: 8 (catálogo).

Diagnose. Sintergoesternito 8 com área membranosa larga estendendo-se do ápice à base (Fig. 61); trocanter posterior com protuberância curta, achatada, semitransversa (Fig.
16); fêmur posterior dilatado com franja de cerdas longas na face posterior (Fig. 15); surstilos cerdosos, assimétricos (Figs. $59,60)$; ápice do guia fálico alongado; bulbo do apódema ejaculador com tubérculos.

Material examinado: ARGENT[ina], Catamarca, L. Nacimiento, 1530.xi.1968, Terán, Willink \& Stange, Malaise, 1 macho (PROIMI); Tucumán, Tafi Del Vale, 18.iv.1994, E. Virla, Malaise, 1 macho (INPA); 03.x.1996, c/ red, D. Kuscheli, 1 macho (PROIMI).

Distribuição geográfica: Argentina (Buenos Aires, Santiago Del Estero, Salta, Catamarca*) e Paraguai.

\section{Tomosvaryella lynchi (Shannon, 1927)}

(Figs. 17, 62-64)

Pipunculus lynchi Shannon, 1927: 38.

Tomosvaryella lynchi; Aczél, 1948: 25 (dist.); 1952: 248 (catálogo); Hardy, 1953: 300, figs. 1-4 (redescrição); 1966: 8 (catálogo); Ale-Rocha \& Rafael, 1995: 409, 413, figs. 14-20 (chave, revisão); De Meyer, 1996: 89 (catálogo).

Diagnose. Protuberância ventral do trocanter posterior com ápice afilado ou arredondado (Fig. 17), surstilos alargados apicalmente (Figs. 62, 63); ápice do guia fálico curto (Fig. 64). 

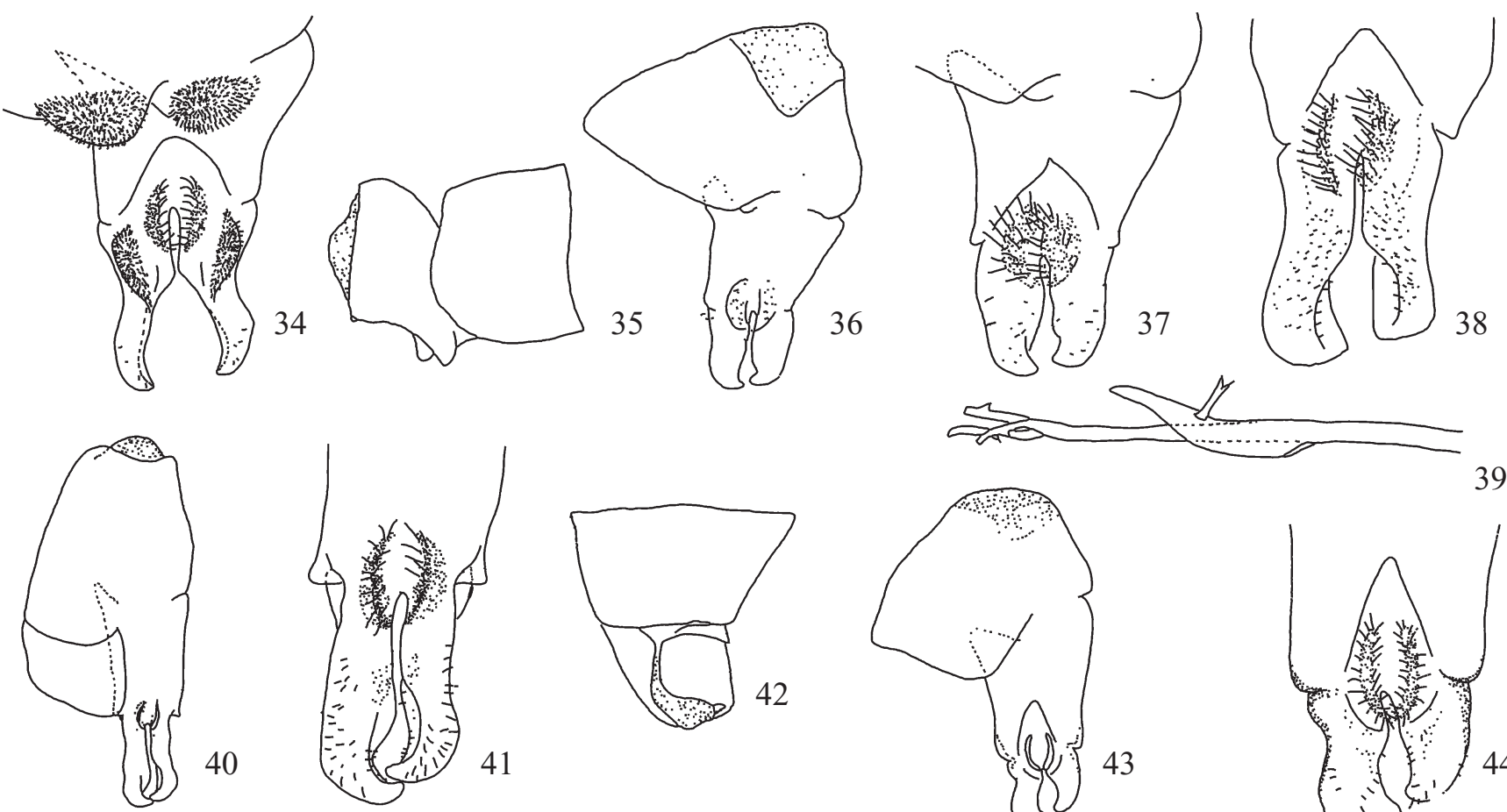

40
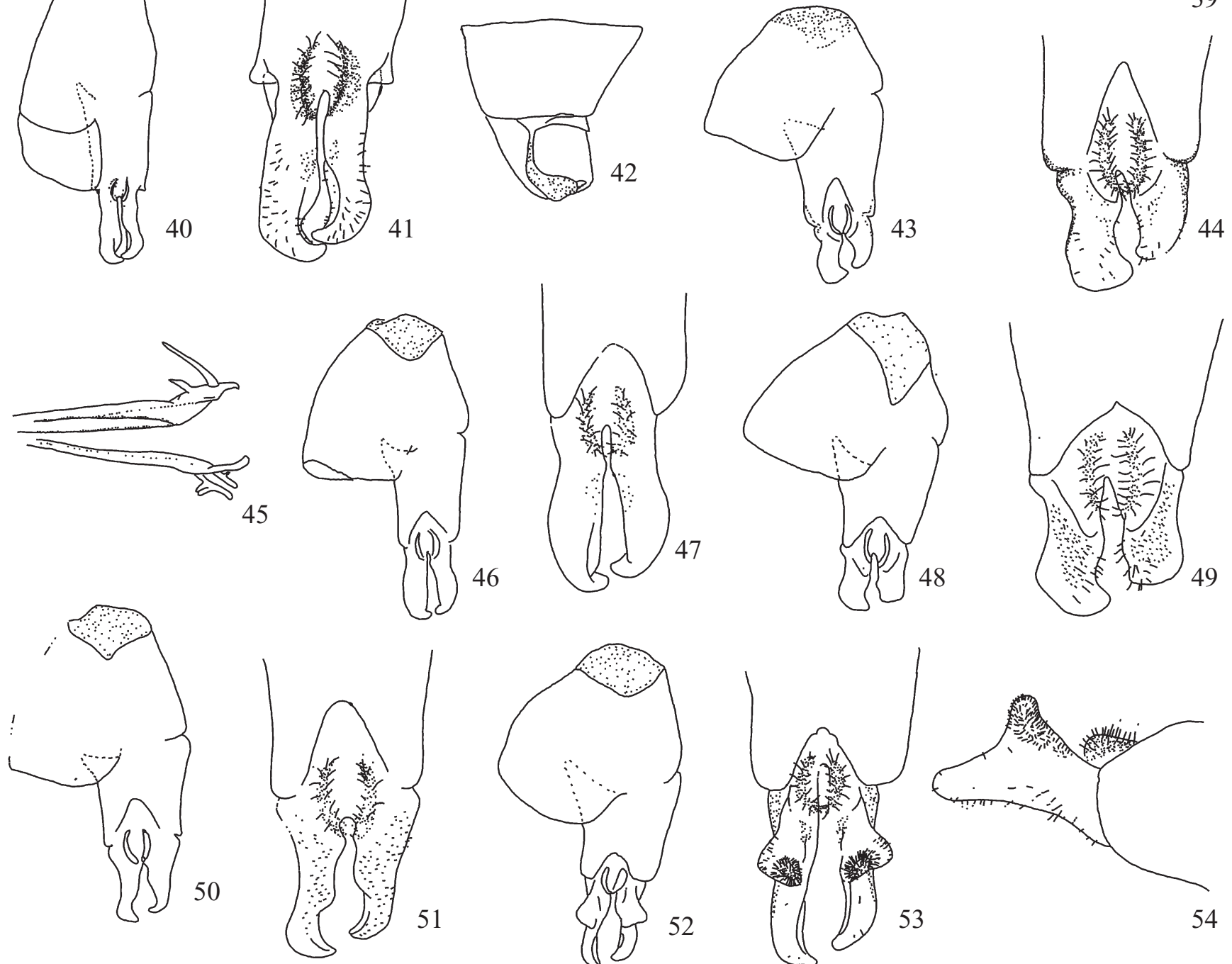

Figs. 34-54. Terminália masculina (Figs. 34, 36-38, 40-41, 43-44, 46-54), sintergoesternito 8 (35, 42), guia fálico e edeago (Figs. 39, 45): 34-35, Tomosvaryella amazonensis; 36-37, T. aurata; 38, T. bidens; 39-42, T. bissulca; 43-45, T. chilensis; 46-47, T. crinita; 48-49, T. curta; 50-51, T. diffusa; 52-54, T. ekyphysis. (Figuras retiradas de Ale-Rocha 1992a, b, 1993, 1996 e Ale-Rocha \& Rafael 1995).

Material examinado: ARGENT[ina], Salta, Yacochuia, Malaise, 0115.x.1968, Terán, Willink \& Stange, 1 macho (PROIMI); 01-15.xii.1970, 1 macho (INPA).

Distribuição geográfica: E.U.A., México, Costa Rica, Brasil e Argentina (Missiones, Tucumán, Salta*).

\section{Tomosvaryella pectinalis Ale-Rocha, 1996}

(Figs. 22, 71-73)

Tomosvaryella pectinalis Ale-Rocha, 1996: 177-178, figs. 100-107; De Meyer \& Skevington, 2000: 8 (catálogo). 


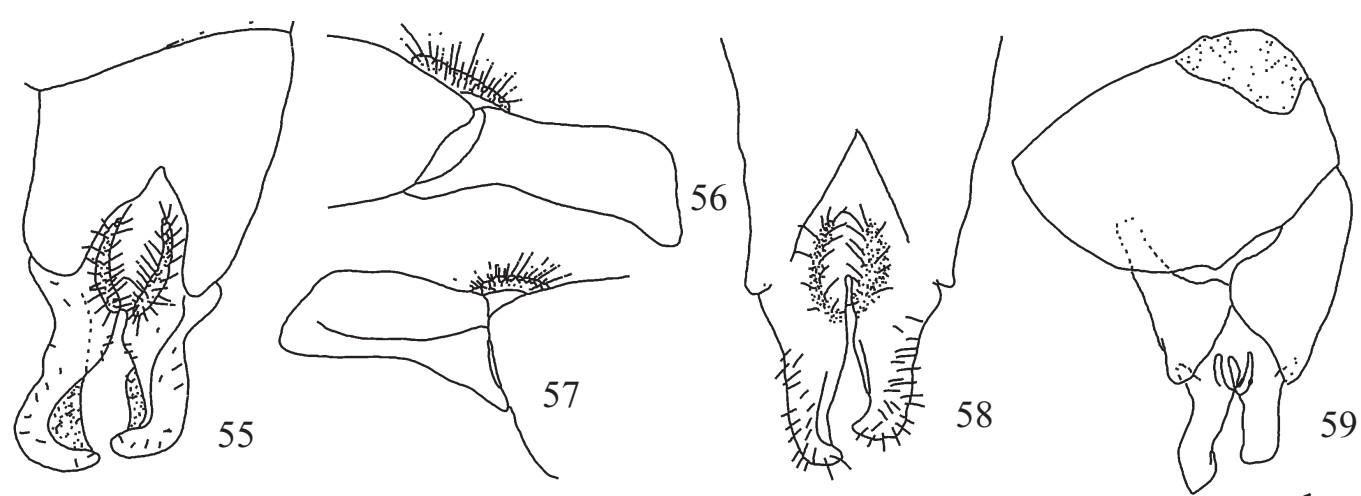

59
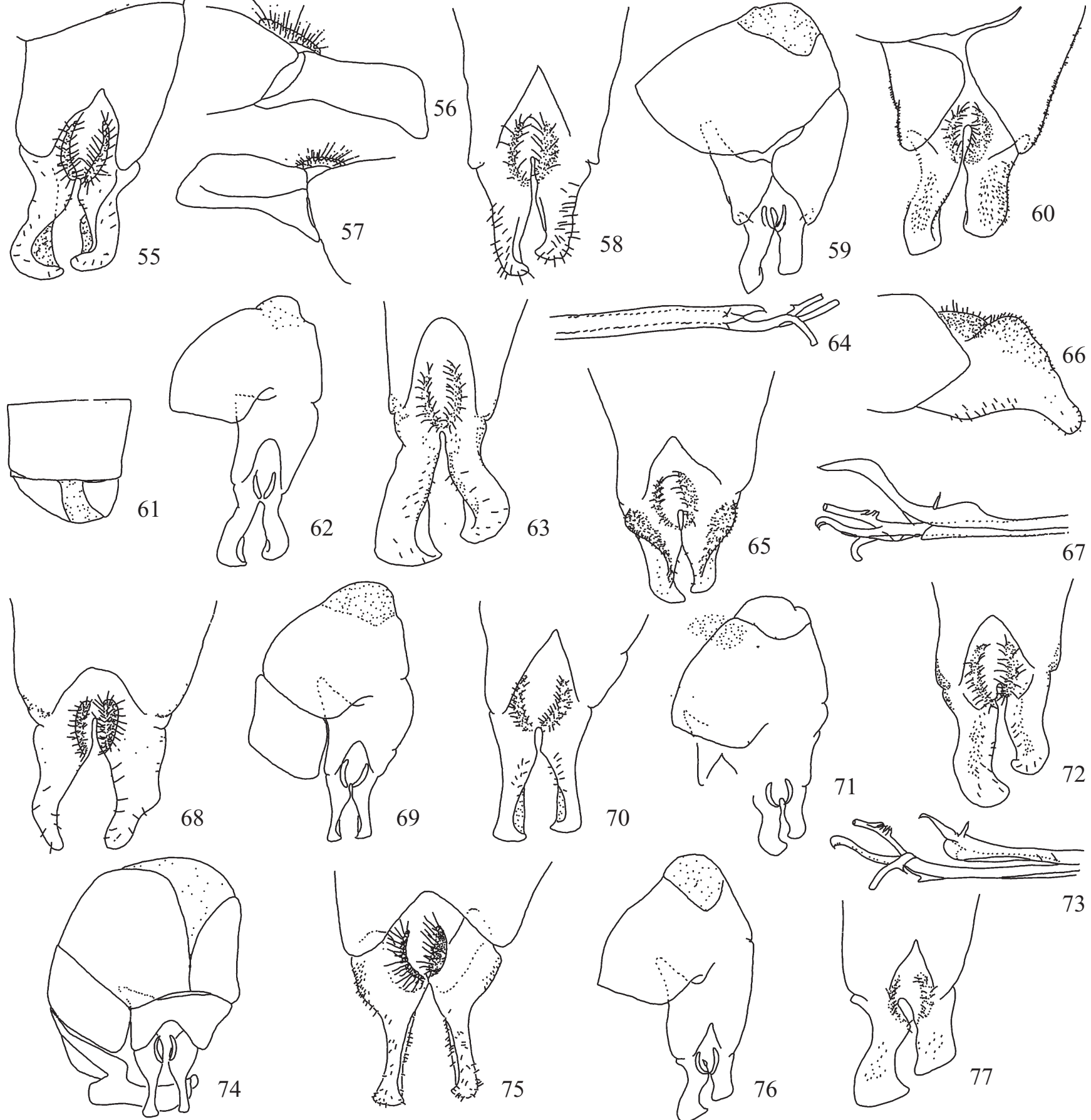

Figs. 55-77. Terminália masculina (Figs. 55-60, 62, 63, 65, 66, 68-72, 74-77), sintergoesternito 8 (Fig. 61) e guia fálico e edeago (Figs. 64, 67, 73): 5557, T. galapagensis; 58, T. incompta; 59-61, T. longiseta; 62-64, T. lynchi; 65-67, T. manauensis; 68, T. mediocris; 69-70, T. ornatitarsalis; 71-73, T. pectinalis; 74-75, T. perissosceles; 76-77, T. platensis. (Figuras retiradas de Ale-Rocha 1992a, b, 1993, 1996 e Ale-Rocha \& Rafael 1995).

Diagnose. Cerdas torácicas alongadas; cerdas alongadas na face posterior do fêmur posterior; trocanter posterior com protuberância ventral curta e arredondada (Fig. 22); surstilos robustos, arredondados apicalmente (Figs. 71, 72); guia fálico com espinho dorsal subapical; ramo dorsal do edeago com pente de espinhos desenvolvidos (Fig. 73).

Material examinado: ARGENT[ina], Catamarca, L. Nacimiento, Malaise, 15-28.ii.1968, Terán, Willink \& Stange, 1 macho (PROIMI).
Distribuição geográfica: Chile e Argentina* (Catamarca).

\section{Tomosvaryella perissosceles Hardy, 1965}

(Figs. 23, 74, 75)

Tomosvaryella perissosceles Hardy, 1965: 238, figs. 16b-f; 1966: 8 (catálogo); Ale-Rocha, 1996: 179, figs. 108-114 (revisão); De Meyer, 1996: 61 (catálogo). 
Diagnose. Tórax com cerdas inconspícuas; trocanter posterior com protuberância espiniforme ventral longa; tíbia posterior arqueada; tarso posterior achatado, $1^{\circ}$ tarsômero mais largo que a tíbia (Fig. 23); surstilos delgados, subsimétricos (Figs. 74, 75).

Material examinado: ARGENT[ina], Catamarca, Belen, Malaise, 0115.iii.1971, Terán, Willimk \& Stange, 1 macho (PROIMI); 01-15.i.1996, 1 macho (INPA); Tucumán, Taf. Del Vale, 30.vi.1995, c/red., E. Virla, 2 machos (PROIMI); 12.v.1995, Malaise, 6 machos (PROIMI); 01.viii.1995, 3 machos (INPA), 01.vi.1996, 5 machos (INPA)

Distribuição geográfica: Argentina (Jujuy, Catamarca*, Tucumán*).

\section{Tomosvaryella platypoda sp. nov.}

(Figs. 105-109)

Diagnose. Trocanter posterior com protuberância ventral mediana, curto com ápice truncado, tarsômeros posteriores alargados; surstilos simétricos e estreitados distalmente.

Descrição. Holótipo macho. Corpo: 3,2 mm. Asa: 3,4 mm. Cabeça: pós-crânio largo, marrom-escuro a preto com pruinosidade cinza; triângulo ocelar marrom-escuro; ocelos marrons; olhos unidos na fronte por distância equivalente a 4-5 facetas; triângulo frontal marrom-escuro a preto, largo, com pruinosidade cinza-prateada; escapo marrom-escuro; pedicelo marrom-escuro com duas cerdas apicais pretas, pequenas; flagelo marrom-claro, mais claro em direção ao ápice, ápice filiforme e curto; face marrom-escura a preta com pruinosidade cinza-prateada, visível a certa luz. Tórax: escuto marrom-escuro a preto com pruinosidade cinza esparsa, mais densa na margem anterior; cerdas amarelas, esparsas, delgadas e curtas; lobo pós-pronotal amarelo com cerdas amarelas; calo pós-alar com uma cerda amarela; escutelo e sub-escutelo marrom-escuros a pretos, pruinosidade cinza no escutelo e cinza-prateada densa no sub-escutelo. Pernas: coxas marrom-escuras a pretas, com ápices amarelos e pruinosidade cinza esparsa; coxa média com cerdas castanhas longas, conspícuas no ápice; trocanteres marrons, o posterior mais claro no ápice e com protuberância ventral curta com ápice truncado (Fig. 108); fêmur anterior e médio marrons com ápice amarelo e ctenídios ventrais presentes na metade distal do fêmur médio; fêmur posterior marrom com ápice e base amarelos; tíbias marrons com 1/4 basal e ápice amarelos, posterior mais robusta que as demais; tarsos amarelos com o $5^{\circ}$ tarsômero marrom; tarsos anterior e médio delgados com o $1^{\circ}$ tarsômero duas vezes mais longo que o segundo; tarso posterior (Fig. 109) alargado. Asa: hialina com iridescência dourada; $3^{\mathrm{a}}$ seção costal metade do comprimento da $4^{\mathrm{a}}$, lobo anal largo, 1 cerda fraca na base da asa; halter amarelo pálido. Abdômen: ovalado, marrom-escuro com pruinosidade cinza esparsa; tergito 1 com cerdas laterais castanhas e longas, com pruinosidade dourada na base e uma faixa estreita mediana de pruína cinza, visíveis a certa luz; manchas dorso-laterais de pruinosidade cinza-prateada nos segmentos 2-5. Terminália: sintergoesternito 8, em vista dorsal, pouco mais curto que o tergito 5; surstilos subsimétricos com base larga e ápice mais estreito, curvados internamente, tão longos quanto o epândrio (Figs. 105, 106); ramos do edeago discretamente alongados, o dorsal com um espinho robusto (Fig. 107). Guia fálico não visível.

Fêmea. Desconhecida.

Material examinado: Holótipo macho (PROIMI), ARGENT[INA], Catamarca, L., Belen, Malaise, 15-30.xi.1968, Terám Willik \& Strange. Parátipos: ARGENT[INA], Catamarca, Belen, 16-31.x.1968, 6 machos (2 INPA, 4 PROIMI); 15-30.xi.1968, 14 machos (4 INPA, 10 PROIMI); 15 31.xii.1968, 3 machos (1 INPA, 2 PROIMI); 01-15.ix.1969, 2 machos (PROIMI).

Distribuição geográfica: Argentina.

Etimologia: Do grego platys = largo, achatado; podos $=$ pé. Refere-se aos tarsos posteriores achatados.

\section{Tomosvaryella scopulata Hardy, 1963}

\section{(Figs. 27, 86)}

Tomosvaryella scopulata Hardy, 1963:262; 1966: 8 (catálogo); Ale-Rocha \& Rafael, 1995: 409 (revisão); De Meyer, 1996: 93 (catálogo).

Diagnose. Corpo preto, tórax com cerdas curtas e pruinosidade dorsal marrom, mais pálida na região pré-sutural; trocanter posterior com protuberância ventral curta e triangular (Fig. 27); face posterior do fêmur posterior com cerdas delgadas e alongadas; terminália estreita, surstilos robustos com projeção dorsal longitudinal cerdosa e truncados apicalmente (Fig. 86), guia fálico simples, ramificações do edeago curtas, bulbo do apódema ejaculador alargado no ápice.

Material examinado: ARGENT[ina], Tucumán, Malaise, 02.iv.1994, c/ red., E. Virla, 1 macho (PROIMI).

Distribuição geográfica: México, Cuba, Dominica, Colômbia, Argentina* (Tucumán).

\section{Tomosvaryella spangleri Scarbrough \& Knutson, 1989}

(Figs. 28, 87, 88)

Tomosvaryella spangleri Scarbrough \& Knutson, 1989: 526, 530, figs. 610; De Meyer, 1996:94 (catálogo).

Diagnose. Escuto e escutelo com pruinosidade marromavermelhada; trocanter posterior com protuberância ventral curta, afilada e pilosa apicalmente (Fig. 28); surstilos angulosos na base, estreitados medialmente e com os ápices em pinça (Fig. 88); ápice do parâmero longo com espinho dorsal curto; edeago com ramificações médias (Fig. 87).

Material examinado: ARGENTINA, Tucumán, Malaise, 02.iv.1994, c/ red., E. Virla, 1 macho (PROIMI).

Distribuição geográfica: Dominica, Porto Rico, Argentina* (Tucumán). 

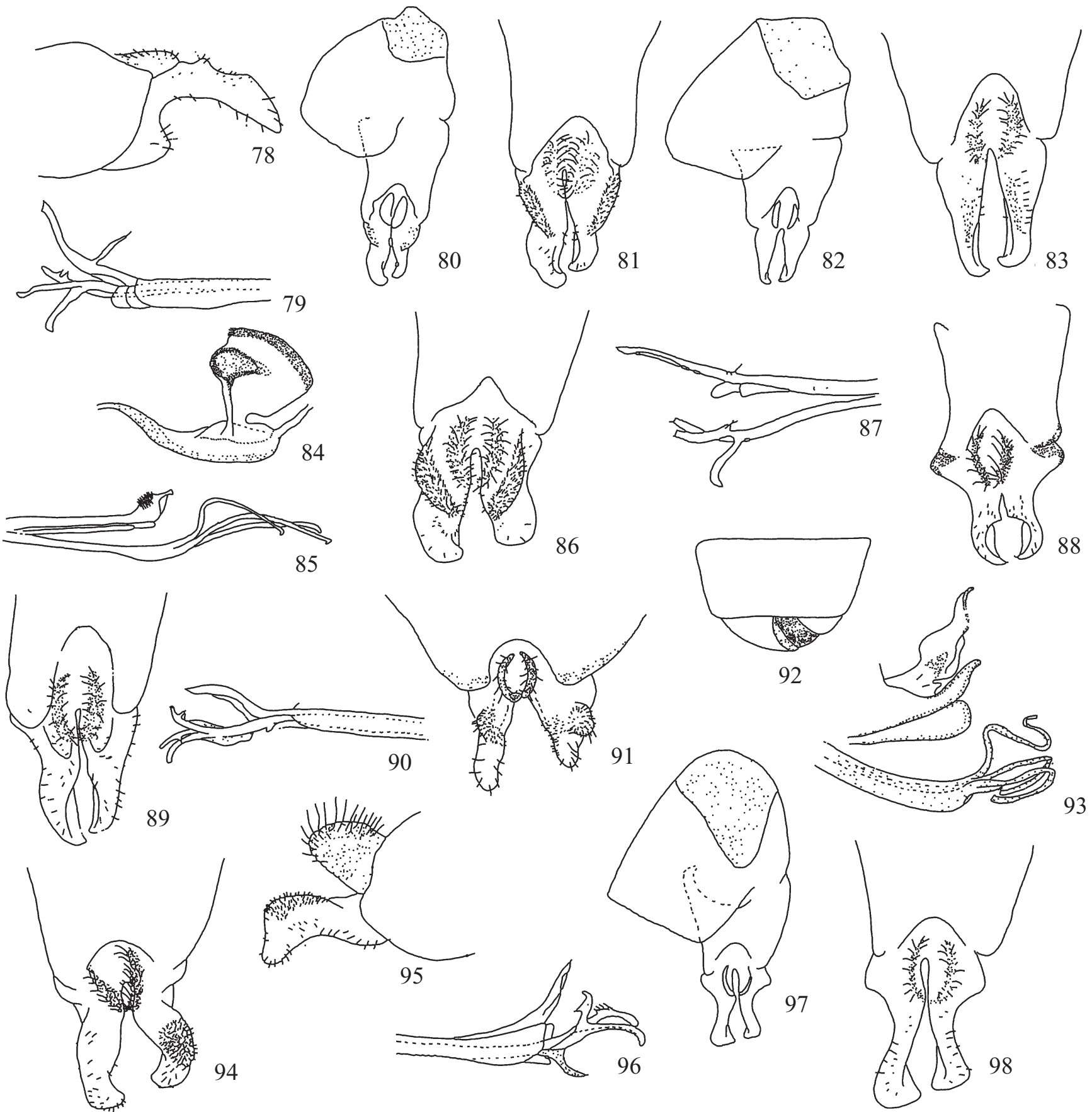

85

86

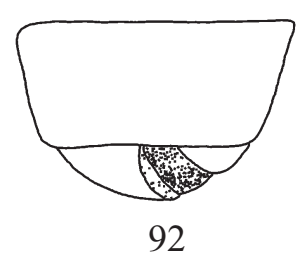

88

Figs. 78-98. Terminália masculina (Figs. 78, 80-83, 86, 88, 89, 91, 94-95, 97-98), sintergoesternito 8 (Fig. 92), guia fálico e edeago (Figs. 79, 85, 87, 90, 93, 96), e apódema ejaculador (Fig. 84): 78-79, T. platensis; 80-81, T. prostata; 82-85, T. santiagoensis; 86, T. scopulata; 87-88, T. spangleri; 89-90, T. spinosa; 91-93, T. subvirescens; 94-96, T. venezuelana; 97-98, T. virlai. (Figuras retiradas de Ale-Rocha 1992a,b, 1993, 1996 e Ale-Rocha \& Rafael 1995).

\section{Tomosvaryella virlai Ale-Rocha, 1996}

(Figs. 32, 33, 97, 98)

Tomosvaryella virlai Ale-Rocha, 1996: 169, figs. 152-158; De Meyer \& Skevington, 2000: 8 (catálogo).

Diagnose. Quarto tarsômero anterior com projeção lobular na face ventral (Fig. 33); troncanter posterior com protuberância ventral cônica (Fig. 32); surstilos subsimétricos, angulosos na base e estreitados medialmente (Figs. 97, 98); ramo dorsal do edeago com série de espinhos delgados, ápice do guia fálico curto e simples.

Material examinado: ARGENTINA, Canuela, Trampa, Malaise, ix.1994-v.1995, Terán, Willink \& Stange, 1 macho (INPA).

Distribuição geográfica: Argentina (Buenos Aires, Missiones). 

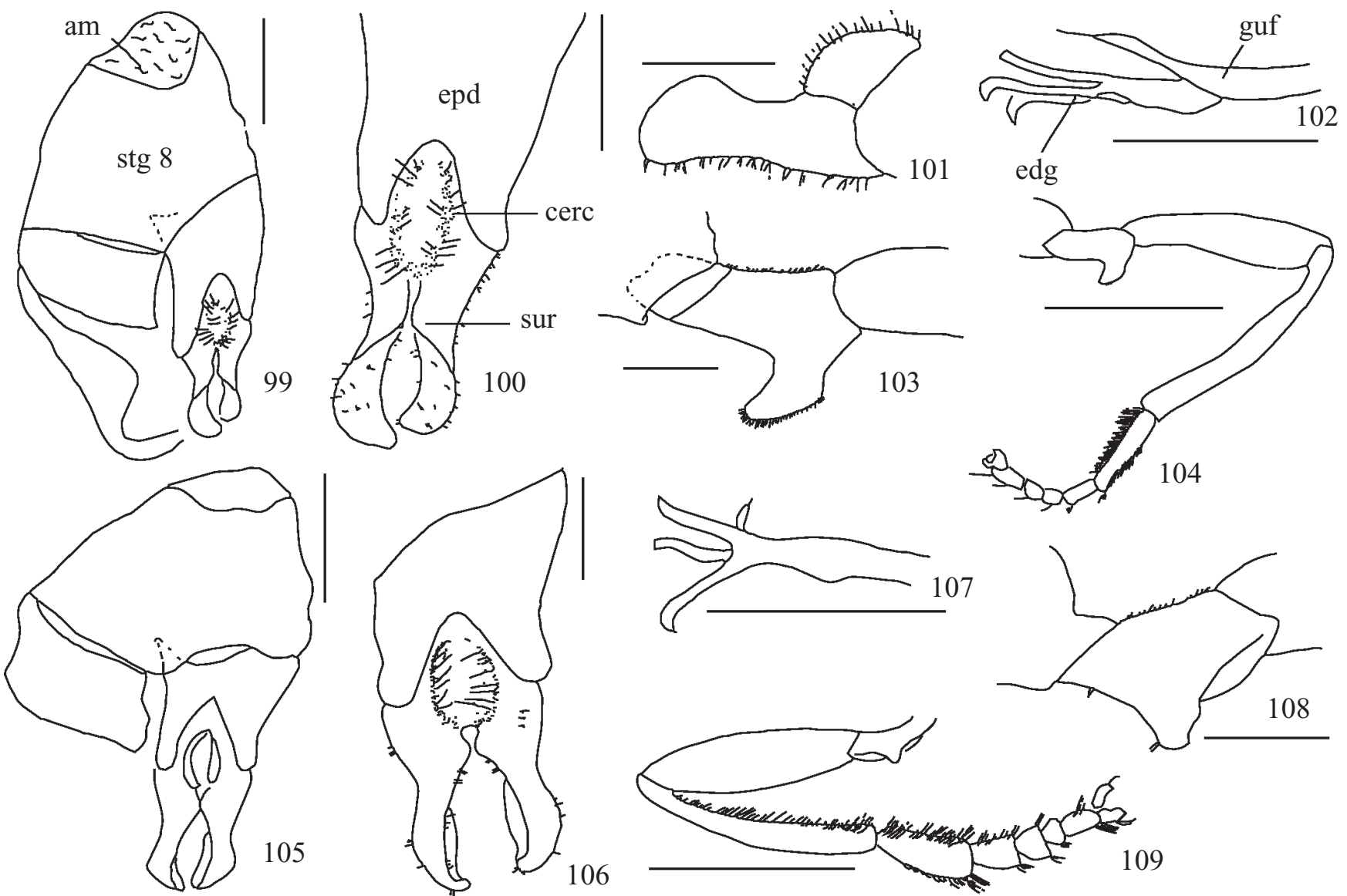

Figs. 99-109. Tomosvaryella lobata sp. nov. (parátipo) (Figs. 99-104) e Tomosvaryella platypoda sp. nov. (parátipo) (Figs. 105-109): 99-101, 105-106, terminália masculina externa; 102, 107, guia fálico e edeago; 103, 108, trocanter posterior; 104, 109, perna posterior. (Escalas: 99-102, 105-106, 107 = $0,1 \mathrm{~mm} ; 103,108=0,2 \mathrm{~mm} ; 104,109=0,5 \mathrm{~mm}$ ) (Abreviaturas: am, área membranosa; cerc, cerco; edg, edeago; epd, epândrio; guf, guia fálico; stg, sintergoesternito; sur, surstilo).

\section{Chave de identificação para as espécies sul-americanas de Tomosvaryella (machos)}

1. Sintergoesternito 8 sub-hemisférico (Fig. 92); surstilos tuberculados (Fig. 91); edeago com ramificações longas e enroladas (Fig. 93); trocanter posterior com processo ventral curto e achatado no ápice (Fig. 30) (cosmopolita)

T. subvirescens (Loew)

1 '. Sintergoesternito 8 comprimido para a direita; outra combinação de caracteres

2(1'). Lobo anal desenvolvido; triângulo ocelar longo; olhos contíguos por cerca de 8 facetas; ápice do flagelo filiforme; trocanter posterior com processo ventral arredondado com cerdas fortes (Fig. 19); surstilos assimétricos (Fig. 68) (Chile)

T. mediocris (Collin)

2. Lobo anal reduzido; outra combinação de caracteres ... 3

$3\left(2^{\prime}\right)$. Trocanter posterior com protuberância ventral espiniforme, longo ou curto, com ápice afilado ou arredondado, reto ou curvo (ex. Figs. 1, 2, 4, 5, 20, 25) .......... 4

3 '. Trocanter posterior com protuberância ventral curta, de forma variada, geralmente arredondada, não espiniforme (ex. Figs. 6, 10, 13, 16, 18) .
4(3). Trocanter posterior com duas protuberâncias em forma de espinho (Figs. 1, 2, 5)

4'. Trocanter posterior com uma protuberância em forma de espinho (Figs. 4, 20, 23, 25) ....

\section{6}

5(4). Processos espiniformes do trocanter posterior longos, subiguais em comprimento (Fig. 5); surstilos subsimétricos e robustos (Fig. 38); sintergoesternito 8 normal (EUA, México, El Salvador, Bahamas, Brasil)

T. bidens (Cresson)

5'. Protuberâncias espiniformes do trocanter posterior curtas, uma mediana e outra mais curta na margem distal, às vezes perpendicular à mediana (Figs. 1, 2); sintergoesternito 8 com dois tubérculos na margem distal (Fig. 35); surstilos com tuberosidade cerdosa na base (Fig. 34) (Brasil, Chile, Paraguai, Argentina)

\section{T. amazonensis De Meyer \& Skevington}

$6\left(4^{\prime}\right)$. Tarsos posteriores modificados, alargados ou com formas variadas. 7

6'. Tarsos posteriores simples, delgados ...... 9

7(6). Primeiro tarsômero do tarso posterior longo, mais de três vezes mais longo que o segundo tarsômero; protube- 
rância ventral do trocanter longa e delgada; surstilos quase retos

7'. Primeiro tarsômero do tarso posterior curto, duas vezes mais longo que o segundo tarsômero (Fig. 109); protuberância ventral do trocanter curta e robusta (Fig. 108); surstilos curvados internamente (Fig. 106) (Argentina) .

\section{T. platypoda sp. nov.}

8 (7). Tarsos posteriores com projeções ventrais lobulares (Fig. 21); trocanter posterior como na Fig. 20; surstilos subsimétricos, delgados em vista ventral (Figs. 69, 70) e robustos em vista lateral, com ápices truncados (Brasil)....

T. ornatitarsalis Hardy

8'. Tarsos posteriores achatados, primeiro tarsômero mais largo que a tíbia, tíbia posterior arqueada (Fig. 23); surstilos subsimétricos e estreitos (Figs. 74, 75) (Argentina) .......

T. perissosceles Hardy

9(6'). Surstilos alargados apicalmente (como nas Figs. 63, 100)

... 10

9'. Surstilos não alargados apicalmente (como nas Figs. 36, $37,80,81)$ 11

10(9). Ápice do guia fálico curto (Fig. 64), surstilo constrito na base (Figs. 62, 63) (México, Bahamas, Costa Rica, Cuba, República Dominicana, Haiti, Jamaica, Brasil, Peru, Argentina)

T. lynchi (Shannon)

10'. Ápice do guia fálico longo (Fig. 102), surstilo constrito na porção mediana (Figs. 99, 100) (Argentina)

T. lobata sp. nov.

11(9'). Processo espiniforme do trocanter delgado (Fig. 4); quinto tarsômero anterior com pequena projeção basal, ventralmente (Fig. 3); catatergito com forte pruinosidade dourada; surstilos curtos e robustos sem projeção lateral longitudinal cerdosa (Figs. 36, 37) (Brasil, Argentina) ..

T. aurata Ale-Rocha

11'. Processo do trocanter robusto (Fig. 25); quinto tarsômero anterior sem modificação; catatergito com pruinosidade cinza-prateada; surstilos alongados com projeção lateral longitudinal cerdosa (Figs. 80, 81) (Colômbia)

T. prostata Hardy

12(3'). Trocanter posterior com espinhos ventrais fortes (Fig. 29); surstilos subsimétricos, estreitados apicalmente (Fig. 89); guia fálico com ápice longo (Fig. 90) (Argentina) ..

T. spinosa Ale-Rocha

12'. Trocanter posterior sem espinhos fortes, surstilos e guia fálico variáveis .

13. Trocanter posterior com processo curto, semitransverso, truncado apicalmente (Fig. 16); fêmur posterior dilatado, com cerdas longas na face posterior (Fig. 15); surstilos cerdosos, robustos, truncados apicalmente (Figs. 59, 60); sintergoesternito 8 com área membranosa larga dorsalmente (Fig. 61) (Paraguai, Argentina)

T. longiseta Ale-Rocha

13'. Trocanter posterior e fêmur posterior não como acima; surstilos variáveis
14. Tarsos posteriores com cerdas longas e finas na face interna (Fig. 8); surstilos subsimétricos, longos, quase glabros (Figs. 46, 47); bulbo do apódema ejaculador longo e estreitado, às vezes dilatado apicalmente (Argentina) T. crinita Ale-Rocha

14'. Tarsos posteriores sem cerdas longas; outros caracteres variáveis

15. Quarto tarsômero da perna anterior com projeção lobular ventral (Fig. 33); tarsos posteriores achatados e alargados, primeiro tarsômero maior que os três seguintes combinados em comprimento; surstilos subsimétricos, angulosos na base, estreitados medialmente (Figs. 97, 98) (Argentina) T. virlai Ale-Rocha

15'. Quarto tarsômero da perna anterior sem projeção lobular ventral; outros caracteres variáveis 16

16. Tarsômeros posteriores, principalmente o primeiro, dilatados lateralmente (Fig. 11); trocanter posterior com protuberância desenvolvida (Fig. 10); surstilos assimétricos (Figs. 50, 51) (Guiana, Brasil, Argentina)....

T. diffusa Ale-Rocha

16'. Tarsômeros posteriores não dilatados; outros caracteres variáveis 17

17(16’). Cerdas torácicas alongadas; fêmur posterior com cerdas curtas ou longas na face posterior ................... 18

17'. Cerdas torácicas curtas, frequentemente inconspícuas; fêmur posterior com cerdas longas na face posterior . 23

18(17). Tórax com faixas longitudinais de pruinosidade cinza-prateada e marrom; tergitos abdominais com manchas laterais de pruinosidade cinza-prateada densa; surstilos subsimétricos (Figs. 82, 83); bulbo do apódema ejaculador arredondado (Fig. 84); ramificações do edeago longas (Fig. 85); trocanter posterior como na Fig. 26 (Chile) ....

T. santiagoensis De Meyer \& Skevington

18'. Tórax não como acima; manchas de pruinosidade no abdome, quando presentes, restritas a alguns tergitos; outros caracteres variáveis .

19(18'). Sintergoesternito 8 alongado e estreitado (Figs. 40, 42); tarsos castanhos dorsalmente com a face ventral amarela ou amarelos, com o último tarsômero castanho; surstilos longos, ápices côncavos internamente (Fig. 41); guia fálico com espinho dorsal bifurcado (Fig. 39) (Chile, Argentina) T. bissulca Ale-Rocha

19'. Sintergoesternito 8 curto; tarsos castanho-claros a amarelos; surstilos e guia fálico variáveis 20

20. Ápice do guia fálico longo, ultrapassando o comprimento dos ramos do edeago (ex. Fig. 79); surstilo direito largo e truncado apicalmente ............................................... 21

20'. Ápice do guia fálico curto, não ultrapassando o comprimento dos ramos do edeago (ex. Figs. 45, 73); surstilo direito estreito e arredondado apicalmente ................ 22

21(20). Surstilo esquerdo fortemente côncavo em vista lateral (Fig. 78), distintamente mais longo que o direito (Figs. 
76, 77); guia fálico com espinho dorsal longo (Fig. 79); trocanter posterior como na Fig. 24 (Argentina)

T. platensis Ale-Rocha

21'. Surstilo esquerdo apenas levemente arqueado em vista lateral, surstilos curtos (Figs. 48, 49); guia fálico sem espinho dorsal longo (Chile, Argentina)

T. curta Ale-Rocha

22(20’). Surstilos mais curtos que o epândrio (Figs. 43, 44); guia fálico com dois espinhos dorsais fortes, o anterior cerca de três vezes o comprimento do posterior (Fig. 45); trocanter posterior como na Fig. 7 (Chile).

T. chilensis Ale-Rocha

22'. Surstilos tão ou mais longos que o epândrio (Figs. 71, 72); guia fálico com um espinho dorsal curto e delgado, ramo dorsal do edeago com pente de espinhos desenvolvidos (Fig. 73) (Chile, Argentina)

\section{T. pectinalis Ale-Rocha}

$23\left(17^{\prime}\right)$. Surstilos com protuberância dorsal cerdosa ..... 24

23'. Surstilos sem protuberância dorsal cerdosa, se protuberantes em vista lateral, não cerdosos 27

24(23). Trocanter posterior piloso, levemente côncavo ventralmente (Fig. 18); surstilos estreitados apicalmente com protuberância lateral longitudinal cerdosa (Figs. 65, 66), guia fálico com ápice longo (Figs. 67) (Brasil)

T. manauensis Ale-Rocha

24'. Trocanter posterior abaulado ventralmente; forma dos surstilos e ápice do guia fálico variáveis

25

25(24'). Surstilos assimétricos, o externo com protuberância dorsal cerdosa, o interno quase glabro (Fig. 94) e mais delgado em vista lateral; cercos grandes e porrectos (Fig. 95); um dos ramos do edeago com pente de espinhos desenvolvidos (Fig. 96); trocanter posterior como na Fig. 31 (Venezuela) .......................... T. venezuelana Ale-Rocha

$25^{\prime}$. Surstilos subsimétricos, cercos não como acima, edeago variável 26

26(25'). Surstilos longos, delgados, ápices afilados e côncavos internamente, protuberância dorsal cerdosa e acuminada (Figs. 52-54); trocanter posterior como na Fig. 12 (Brasil) T. ekyphysis Ale-Rocha

26'. Surstilos curtos, robustos, ápices truncados, protuberância dorsal cerdosa arredondada (Fig. 86) (México, Cuba, Dominica, Colômbia, Argentina) ....... T. scopulata Hardy

27(23'). Ápice do guia fálico curto (ex. Fig. 64) ............. 28 27'. Ápice do guia fálico longo (ex. Fig. 87) 29

28(27). Surstilos delgados com cerdas fortes, ápices afilados (Fig. 58); trocanter posterior como na Fig. 14 (Brasil) ...

T. incompta Ale-Rocha

28'. Surstilos robustos com cerdas fracas, ápices alargados (Figs. 62, 63) (E.U.A., México, Costa Rica, Brasil, Argentina) T. lynchi (Shannon)

29(27'). Surstilos com ápices em pinça, com ângulos pronunciados na lateral basal (Fig. 88) (Dominica, Porto Rico,
Argentina) T. spangleri Scarbrough \& Knutson 29’. Surstilos robustos, ápices truncados em vista lateral (Figs. 55-57); trocanter posterior como na Fig. 13 (Ecuador).. T. galapagensis (Curran)

\section{AGRADECIMENTOS}

Ao Dr. Eduardo G. Virla do PROIMI, Tucumán, Argentina, pelo empréstimo de grande parte do material utilizado no estudo. Ao Conselho Nacional de Desenvolvimento Científico e Tecnológico - CNPq e Fundação de Amparo a Pesquisa do Estado do Amazonas - FAPEAM, pelas bolsas concedidas.

\section{REFERÊNCIAS}

Aczél, M. 1948. Grundlagen einer Monographie der Dorilaiden (Diptera). Dorilaiden Studien VI. Acta Zoologica Lilloana 6: 5-168.

Aczél, M. 1952. Catalogo de la família Dorilaidae (Pipunculidae) de la Región Neotropical. Revista de la Sociedad Entomológica Argentina 15: $237-251$.

Ale-Rocha, R. 1992a. Descrição de Tomosvaryella pulchra sp. nom. nov. (Diptera, Pipunculidae). Boletim do Museu Paraense Emílio Goeldi, série Zoologia 8 (2): 315-318.

Ale-Rocha, R. 1992b. Redescrição de Tomosvaryella galapagensis (Curran, 1934) (Diptera, Pipunculidae). Boletim do Museu Paraense Emílio Goeldi, série Zoologia 8: 319-321.

Ale-Rocha, R. 1993. Redescrição e considerações sobre a distribuição geográfica de Tomosvaryella lepidipes Hardey, 1943 e descrição de Tomosvaryella venezuelana sp. nov. (Diptera, Pipunculidae). Revista Brasileira de Entomologia 37: 263-266.

Ale-Rocha, R. 1996. Revisão das espécies de Tomosvaryella Aczél da América do Sul (Diptera, Pipunculidae). Revista Brasileira de Entomologia 40: 165-187.

Ale-Rocha, R. \& J. A. Rafael. 1995. Tomosvaryella Aczél do México, América Central e Antilhas (Diptera, Pipunculidae). Revista Brasileira de Zoologia 12: 407-427.

De Meyer, M. 1989. Taxonomische, systematische en zoogeografische revisie van de genus groep Cephalops Fallén (Diptera, Pipunculidae). Tese de Doutorado, Universit Antwerpen, $230 \mathrm{p}$

De Meyer, M. 1996. World catalogue of Pipunculidae (Diptera). Institut royal des Sciences naturelles de Belgique, Documents de Travail 86: $1-127$.

De Meyer, M. \& J. H. Skevington. 2000. First addition to the World Catalogue of Pipunculidae (Diptera). Bulletin de I'Institut Royal des Sciences Naturelles de Belgique, Entomologie 70: 5-11.

Hardy, D. E. 1953. Notes on the Shannon Types of Dorilaidae from Argentina (Pipunculidae-Diptera). Acta Zoologica Lilloana 10: 299-306.

Hardy, D. E. 1963. Studies in Pipunculidae (Diptera) of Colombia Proceedings of the Hawaiian Entomological Society 18: 259-266.

Hardy, D. E. 1965. The Pipunculidae of Argentina. Acta Zoologica Lilloana 19: $187-241$.

Hardy, D. E. 1966. Family Pipunculidae (Dorialidae), p. 1-15. In: N. Papavero (ed.). A catalogue of the Diptera of the Americas South of the United States. 45. São Paulo, Museu de Zoologia, Universidade de São Paulo.

Kozánek, M. 1992. Contribution to the pipunculid fauna of Mongolia (Diptera: Pipunculidae). Akitu (Kyoto Entomological Society), New Series 131: 1-16.

McAlpine, J. F. 1981. Morphology and terminology: adults, p.9-63. In: J. F. McAlpine et al. (eds.). Manual of Nearctic Diptera. Ottawa, Research Branch, Agriculture Canada, Monograph n ${ }^{\circ} 27$, vol. 1, vi +457 p.

Rafael, J. A. \& M. De Meyer. 1992. Generic classification of the family Pipunculidae (Diptera): a cladistic analysis. Journal of Natural History 26: $637-658$. 
Scarbrough, A. G. \& L. V. Knutson, 1989. Asilidae, Bombyliidae, Conopidae, and Pipunculidae (Diptera) of Dominica, West Indies. Florida Entomologist 72: 219-537.

Shannon, R. C. 1927. Some new Diptera from Argentina. Revista de la
Sociedad Entomológica Argentina 1: 31-42.

Skevington, J. H. \& D. K. Yeates 2001. Phylogenetic classification of Eudorylini (Diptera: Pipunculidae). Systematic Entomology 26: 421452 .

Recebido 22/6/2010; aceito 5/4/2011

Editor: Silvio Shigueo Nihei 